\title{
Novel Computational Model of the Brain Water Metabolism: Introducing an Interdisciplinary Approach
}

Titovets $\mathrm{E}^{*}$

Department of Neurosurgery of Republican Research and Clinical Center of Neurology and Neurosurgery, Minsk, Belarus

${ }^{*}$ Corresponding author: Titovets E, Department of Neurosurgery of Republican Research and Clinical Center of Neurology and Neurosurgery, Fr. Skoriny Str., 24, Minsk, Belarus, 220114, E-mail: eptitovets@ gmail.com

Citation: Titovets E (2018) Novel Computational Model of the Brain Water Metabolism: Introducing an Interdisciplinary Approach. J Comp Biol Sys 2(1): 103

\begin{abstract}
Brain water metabolism ensures the processes of cellular communication, transit of the signaling molecules, neurotransmitters, cytokines and substrates, participates in the clearance of pathogenic metabolites. Many neurological conditions that present serious clinical problems arise from altered fluid flow (e.g. Alzheimer's disease, idiopathic normal pressure hydrocephalus, migraine, traumatic brain injury and stroke). At present, the orthodox theory fails to explain the accumulated experimental evidence and clinical data on the brain water metabolism. Modeling becomes an important approach to testing current theories and developing new working mechanisms.

A novel computational model of brain water metabolism has been developed and explored. Using an interdisciplinary approach the long-recognized nanodimentionality of the brain interstitial space is now viewed as a nanofluidic domain with the fluid flow there governed by the slip-flow principles of nanofluidics. Aquaporin-4 (AQP4) of the astrocyte endfeet membranes ensures kinetic control over water movement across the blood-brain barrier. The pulsatory intracranial pressure presents the driving force behind the transcapillary water flow. The model demonstrates good predictability in respect to some physiological features of brain water metabolism and relevance in explaining clinical conditions. The model may find its use in neurobiological research, development of the AQP4-targeted drug therapy, optimization of the intrathecal drug delivery to the brain tumours, in a research on a broad spectrum of water-metabolic-disorder-related conditions.
\end{abstract}

Keywords: Brain water metabolism; Computational model; Nanofluidics; AQP4-targeted therapy

\section{Introduction}

The brain interstitial and the cerebrospinal fluids are involved in the nutrient and gas transport, non-synaptic intercellular communication (volume transmission), signal transduction, transport and targeted delivery of drugs and metabolites, ionic homeostasis, formation and resolution of the brain $\beta$-amyloid deposits, the migration of cells (malignant cells, stem cells), transfer of heat generated by neuractivity $[1,2]$.

Developed over a century ago the classic concept of the cerebrospinal fluid (CSF) movement asserts that the choroid plexus is an exclusive site of the CSF secretion with the fluid flowing on to the subarachnoid space to sink finally in the sagittal sinus [3]. The new knowledge arising from clinical, molecular, membrane and cellular biology research challenges the conventional concept. The novel insights direct attention to the importance of convectional fluid exchange at the microvascular level throughout entire brain parenchyma, the involvement of the cardiac-cycle-dependent oscillations of the intracranial pressure, highlight the important role of AQP4 in brain fluid homeostasis [3-9].

Brain water turnover at the microvascular level might be as high as of $600 \mathrm{~L}$ per day [10]. Filtration and reabsorption of water at the microvessel level involves all the perivascular, the interstitial and the subarachnoid spaces. The volumetric exchange rate of water may vary from 0.35 to $520 \mathrm{ml} / \mathrm{min}$ [11]. The flow of this magnitude presents a problem for any potential water metabolism mechanism that would have to account for it. 
The width of the interstitial space that typically occupies $20 \%$ of the brain volume is in the range 20-64 nm [12]. From this fact, an opinion has been born and commonly accepted in medical community that the narrowness of the spaces between the cells within the neuropil and the tortuosity of the interstitial space will not permit any significant fluid flow there [13]. Diffusion has been considered a dominant governing mechanism there with the conventional wisdom maintaining that the interstitial space presents a diffusion barrier to fluid movement [13-17].

An interdisciplinary approach makes it possible to view the issue in a different light. The nanodimentionality of the interstitial space suggests a nanofluidic approach to the interstitial space hydrodynamics. Nanofluidics deals with the behaviour of fluids confined to nanoslits, nanochannels, nanopores, etc., where at least one geometrical dimension is in the nanometre scale, usually in the range of 1-100 nm [18]. Surface effects in nanoconfined fluids dominate over the bulk-water properties while the fluids exhibit physical behaviour not observed in larger structures, such as those of micrometre dimensions and above [19] .

A special feature of water movement in nanodimentional spaces manifests itself in significant enhancement of the fluid flux due to the surface hydrodynamic slip [20,21]. Thus aquaporins, the water-conducting nanopores, exhibit water permeability typically three orders of magnitude higher than expected from the classical no-slip framework for the same pore size [22]. From the conventional point of view, this seems counterintuitive and even unsupported when using the no-slip Darcy or Hagen-Poiseuille's equations, as has been done so far to model fluid movement in the brain $[23,24]$.

Recently, there appeared a research paper on monitoring the state of water in the brain extracellular space. On injecting singlewalled carbon nanotubes into the rat brain and using super-resolution imaging, the researchers observed the rheology in live brain interstitial space down to $40 \mathrm{~nm}$. It is the very first direct visualization of the state of nanowater in the interstitial space of the live brain [25]. An interdisciplinary approach to the rheology in the brain interstitial space makes it possible to conceptualize the brain interstitial space as a functional nanofluidic domain where water movement is governed by the slip-flow mechanism.

AQP4 plays a crucial role in the control of the interstitial fluid flow. Abundantly expressed in the astrocyte endfeet membranes enveloping the capillaries, the nanofluidic AQP4 channel presents a kinetically limiting step in the water exchange across the blood-brain barrier (BBB) and water movement in the brain [26-34].

Pulsatility is a feature intrinsic to the functioning of the brain. Within its rigid confinement, there are generated waves of pulsating pressure related to the heart activity. The intracranial pressure pulses propagate through the brain at the speed of sound with their dynamics being identical over the brain parenchyma [35-37]. The osmotic pressure in the blood, the interstitial fluid (ISF) and the cerebrospinal fluid (CSF) is identical and, under physiological conditions, there is isosmotic movement of water from blood into brain tissue. This has been demonstrated in animal experiments with the injection of the tritiated water into the systemic blood $[26,38]$. The pulsatile intracranial pressure gradients present a driving force behind the isosmotic flow of the brain fluids.

The aim of the ongoing paper has been to develop a computational model for brain water exchange at the capillary level based on the new knowledge and research information. The model should be able to demonstrate good predictability and account for some pertinent experimental and clinical observations on water metabolism in health and disease.

\section{Methods}

Theoretical and experiment-based assumptions of the model:

-the brain nanodimentional interstitial space presents a nanofluidic domain with the fluid movement there governed by the slipflow mechanism $[18,25]$;

-aquaporin AQP4 ensures kinetic control over water movement between the blood and the brain interstitial space [26,29,34,39];

-the overall fluid transfer between the capillary blood and the interstitial fluid is isosmotic [26,38];

- the pulsatory intracranial pressure presents a driving force behind the isosmotic fluid exchange between the capillaries and the interstitial space $[35,36,40]$.

A capillary presents a rigid-walled cylinder with Poiseuille's viscous laminar flow of Newtonian fluid. A modified phenomenological fluid flux equation, based on the Kedem-Katchalsky formalism of irreversible thermodynamics [41], has been developed to describe water movement between the capillary blood and the brain interstitial space:

$$
J_{v}=L_{p}\left(p_{a}-\Delta p x-f(t)-\pi_{c}-\pi_{I S F}\right)
$$

where $J v\left(\mathrm{~cm}^{3} / \mathrm{s}\right)$ is the volumetric flow rate per unit transfer area $\left(\mathrm{cm}^{2}\right) . L_{p}\left(\mathrm{~cm} / \mathrm{s} / \mathrm{mmHg}\right.$ per sm $\left.{ }^{2}\right)$ is a compound hydraulic conductivity coefficient: 


$$
L_{p}=L_{p}^{A Q P 4}+L_{p}^{*}
$$

where is $L_{p}^{A Q P 4}$ the hydraulic conductivity coefficient associated with AQP4 and $L_{p}^{*}$ is the hydraulic conductivity coefficient of possible other parallel water transfer pathways. Within the context of the ongoing work, we assume that $L_{p}^{A Q P 4} \gg L_{p}^{*}$ from which it follows that $L_{p} \approx L_{P}^{A Q P 4}$.

The hydraulic conductivity coefficient for the astrocyte endfeet membrane expressing AQP4 has been calculated using the data on the water permeability coefficient $\left(P_{f}\right)$ and AQP4 specific density in the astrocyte endfeet membranes by conversion $P_{f}$ to $L_{p}^{A Q P 4}$. The AQP4-dependent hydraulic conductivity coefficient thus obtained is $L_{p}^{A Q P 4}=8.05 \times 10^{-6} \mathrm{~cm} \mathrm{mmHg}^{-1} \mathrm{~s}^{-1}\left(\mathrm{per} \mathrm{cm}^{2} \mathrm{transfer}^{p} \operatorname{area}^{2}\right.$

(for details, see Online Resource, 1)

The capillary longitudinal hydrostatic pressure drops linearly from the arterial to the venous end. The axial hydrostatic pressure gradient, $\Delta p(\mathrm{mmHg} / \mathrm{cm})$, is:

$$
\Delta p=\left(p_{a}-p_{v}\right) / L
$$

where $p_{a}(\mathrm{mmHg})$ and $p_{v}(\mathrm{mmHg})$ is the hydrostatic pressure at the arterial and the venous ends of the capillary, respectively, and $L(\mathrm{~cm})$ is the length of the capillary. The longitudinal distance at any point of the capillary axis, starting from the arterial end, is $x$ $(\mathrm{cm})$.

Simulation of the effect of the capillary length on radial water movement have been carried out by reducing the initial length $L$ of the capillary to the length $L^{*}$ from either the arterial or the venous end. The value of a new hydrostatic pressure $p^{*}$ at the reduced side of the capillary has been estimated from the expression:

$$
p^{*}=p+\Delta p\left(L-L^{*}\right)
$$

It is assumed that no fluid exchange occurs outside $L^{*}, \mathrm{AQP} 4$ distribution is homogeneous and all other parameters of the model are kept constant.

The intracranial pressure as a function of time (the pulse waveform) is $f(t) ; \pi_{c}(\mathrm{mmHg})$ is the capillary plasma oncotic pressure and $\pi_{I S F}(\mathrm{mmHg})$ is the interstitial fluid oncotic pressure.

The volume of water, $J_{h c}$, transferred over a specified period of the heart cycle and a cross-section area is found through numerical integration:

$$
J_{h c}=L_{p}^{A Q P 4} \int_{x_{1}}^{x_{2}} d x \int_{t_{1}}^{f(x)} F(x, t) d t
$$

The model has been developed, using Wolfram Mathematica language, in Wolfram Mathematica 10 program. Parameters and variables for the basic model are given in Online Resource, 3.

\section{Results}

\section{Description of the model}

Figure 1 present simulation results of water movement between the capillary blood and the ISF over a complete cardiac cycle. The heart rate as well as the parameters of intracranial pressure pulse wave stay within physiological limits. The water filtration rate (water movement out of the capillary) is positive while the water reabsorption rate is negative.

The simulation results in Figure 1, obtained with the intracranial pressure pulse wave W1 (Figure 1b), demonstrate a complex character of the transcapillary water exchange over the length of the capillary. The water flux is positive in the CFA, negative in the CRA and oscillatory in the COA. The overall water flow is pulsatory and synchronized with the phases of the heart cycle. 

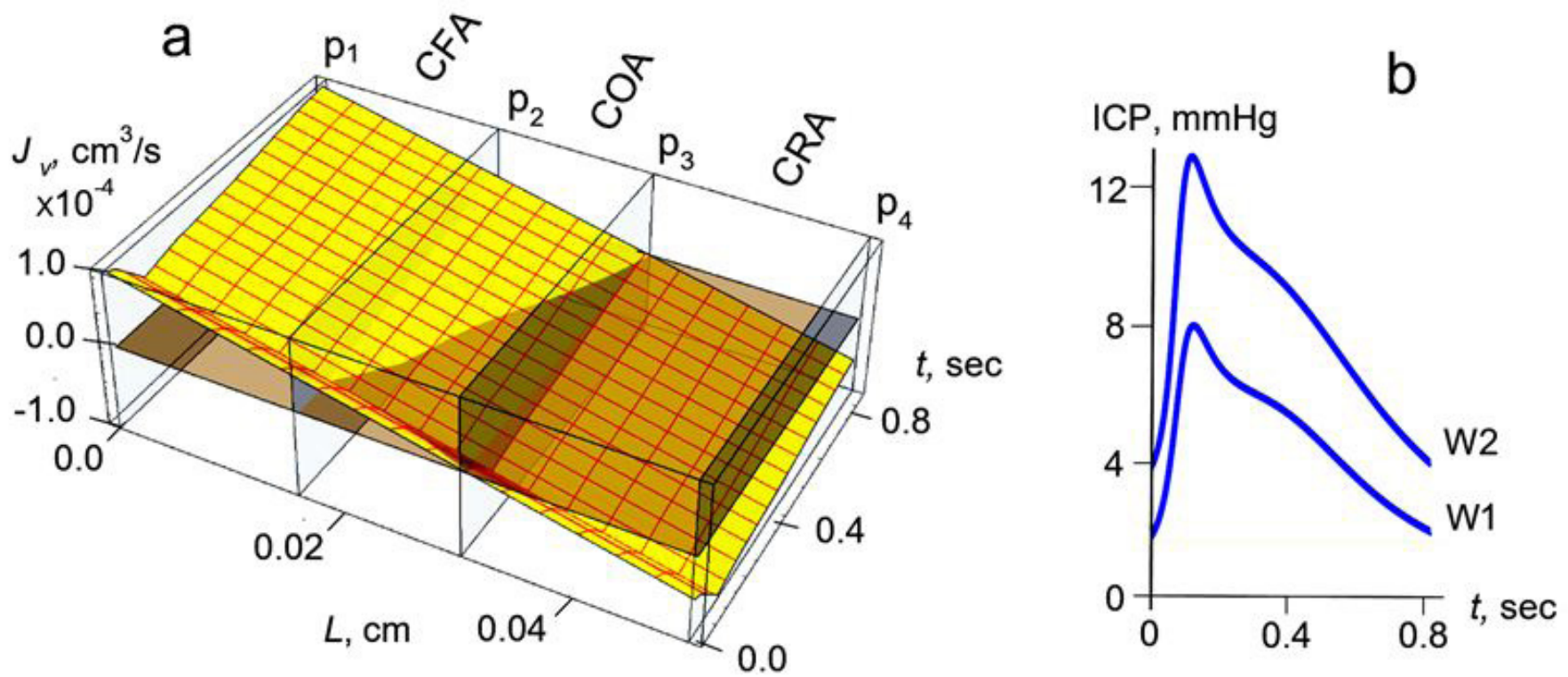

Figure 1: Water exchange between the capillary blood and the ISF after the nanofluidic mechanism

(a) The 3D model of fluid exchange between the capillary blood and the ISF. The infinite planes p1, p2, p3, and p4 delineate different functional parts along the length of the capillary. CFA is the capillary filtration area; COA is the capillary oscillatory area and CRA is the capillary reabsorption area.

(b) The two generic intracranial pressure pulse waveforms, W1 and W2, used in the simulations. A complete heart cycle lasts $0.83 \mathrm{~s}$ (Online resource)

The water flow in the COA presents a special interest. An exploded view of the COA (Figure 2) demonstrates the details of water exchange there.

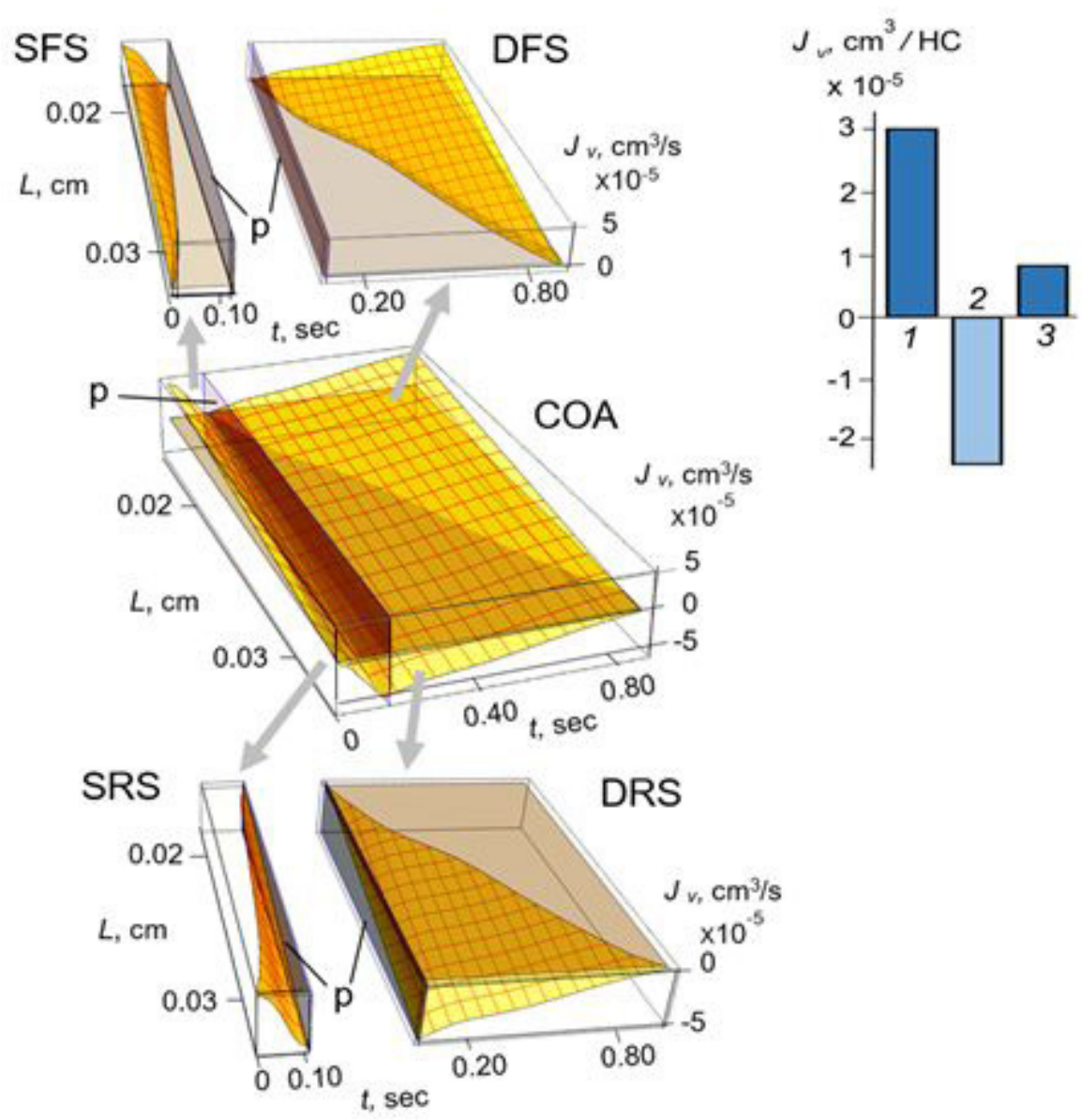

Figure 2: 3D presentation of simulation results of water exchange in the capillary oscillatory area, COA

The infinite plane $p$, passing through the trough in the COA, separates the systolic part of the COA, on the left side of the divide,

from the diastolic part, situated on the right side of the infinite plane. On the time axis, the systolic and the diastolic parts of the COA have coordinates $0 \leq \mathrm{t} \leq 0.12 \mathrm{~s}$ and $0.12 \leq \mathrm{t} \leq 0.83 \mathrm{~s}$, respectively. SFS is the systolic filtration segment; DFS is the diastolic filtration segment; SRS is the systolic reabsorption segment and DRS is the diastolic reabsorption segment. The bar graph shows integral volumes of water, $J_{h c}$ exchanged over one heart cycle. The filtration volume -1 ; the reabsorption volume-2; the net volume-3 
Figure 2 demonstrates the way the ICP pulse wave, on passing over the COA segment, causes the radial flows of water to oscillate between the filtration and the reabsorption functional modes. The volumes of water in each segment have been obtained on numerical integration of the corresponding 3D graphs (Online resource, 4.2-4.5). The bar graph in Figure 2 presents the lumped quantitative data on water movement in the COA. This graph demonstrates an oscillatory pattern of water exchange between the capillary blood and the ISF. Under physiological conditions, the net fluid flux is positive (Bar 3). This oscillating pattern might ensure a feedback exchange mechanism between the blood and the brain, a possibility discussed later in this paper.

The simulation results, combined with the new knowledge make it possible present an outline of the nanofluidic mechanism for the brain water metabolism, Figure 3.

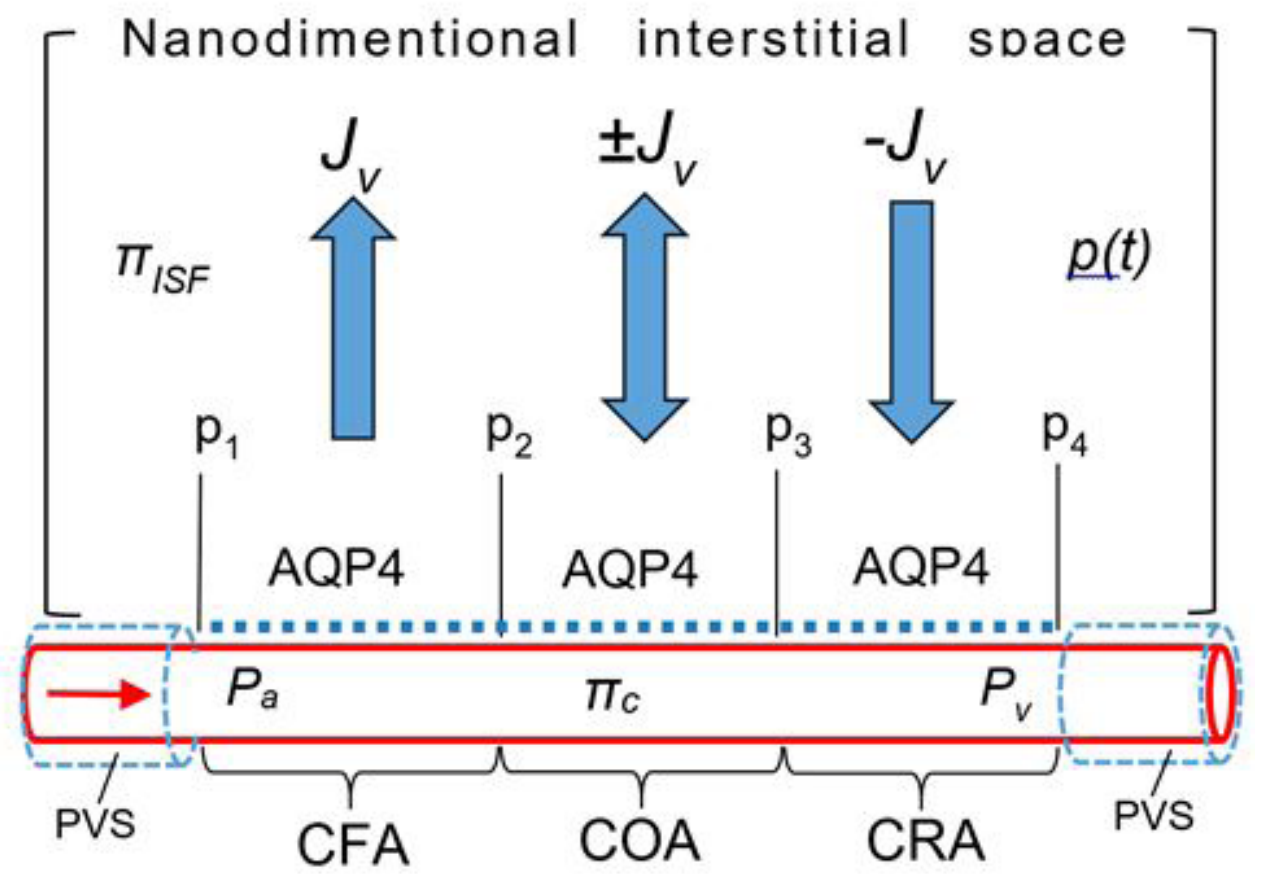

Figure 3: Schematic presentation of the nanofluidic mechanism for the brain water metabolism The red arrow indicate direction of the blood flow in the capillary. The dashed blue line with AQP4 present this aquaporin in the astrocyte endfeet membrane enveloping the capillary. The functional segments of the capillary, similar to the $3 \mathrm{D}$ presentation of the model in Figure 1, are indicated with $\mathrm{p}_{1}, \mathrm{p}_{2}, \mathrm{p}_{3}$, and $\mathrm{p}_{4}$. The blue arrows, with the volumetric flow rate symbols " $J v$ ", “ $\pm J v$ ", and $-J v$ ", indicate the direction of the water flows in the CFA, the COA, and the CRA, respectively. PVS indicates the perivascular spaces. The oncotic pressure in the capillary and the interstitial fluid is $\pi_{C}$ and $\pi_{\mathrm{ISP}}$ respectively. Hydrostatic pressure is $p$ at the arterial end of the capillary and $p_{v}$ at the venular end. The external pulsatory pressure is $p(t)=f(t)$. The square brackets indicate that the water exchange between the capillary and the ISF takes place within the AQP4-controlled nanofluidic domain. Note that the schematic is not drawn to scale and does not represent the true ratio between capillary diameter and the capillary length

\section{Simulation results and predictability of the model}

It is interesting to see how the model would account for some features of brain water metabolism and the effects of various factors influencing it. Among those are the pulsatile nature of the cerebral fluid flow, capillary length and the transcapillary water exchange, the effect of elevated intracranial and venous pressure on the transcapillary water movement, AQP4 lateral polarization in the astrocyte endfeet membrane and the net water flux, to list a few clinically relevant ones. Figure 4 presents the results of the model-computed predictions on the brain water metabolism.

\section{Pulsatile pattern of fluid flow}

Figure 4a,b present the volumetric flow rate profiles in each capillary segment over a few cardiac cycles. A common feature of the flux pattern in each segment is the pulsatile nature of fluid flow synchronized with the phases of the heart cycle. This might seem obvious from the mathematical description of the model. Indeed, the phenomenological fluid flux equation (Eqn.1) includes the intracranial pressure pulse waveform function, $f(t)$, that accounts for the pulsatile pattern of fluid flow. The intracranial pressure pulse waves present a transformation of the heart-driven pulsations of the larger cerebral arteries [37,42]. Pulsatile flow of the CSF may have a more complex nature due to various factors contributing to its particular pattern like a respiratory component, head position etc. In this paper, we consider the cardiac-induced pulsatility only.

\section{Capillary length and the net transcapillary fluid exchange}

The length of brain capillaries lies within a wide range $[43,44]$. Averaged from various geometrically different zones, the mean 

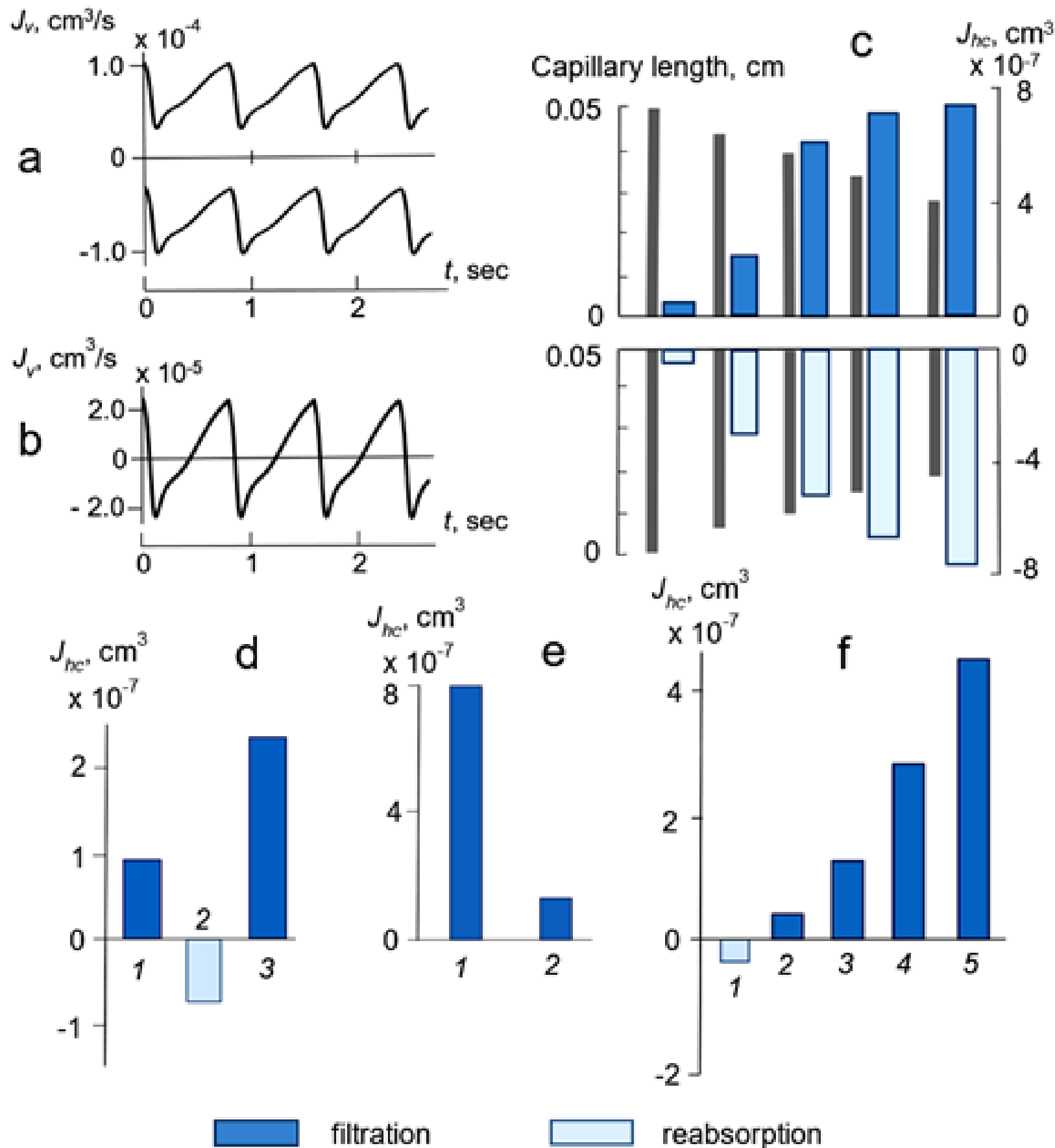

\section{reabsorption}

Figure 4: Simulation results and predictions of the nanofluidic model for the water exchange patterns under specified conditions

(a) Pulsatory patterns of water filtration in the CFA and water reabsorption in the CRA. (b) Oscillatory water flow pattern in the COA. (c) Fluid exchange with the capillaries of different length; $J_{h c}$-water volume transferred over a complete heart cycle. (d) Effect of altered AQP4 lateral polarization in the astrocyte endfeet membrane on the overall capillary water exchange:

Bar 1. AQP4 distributed homogeneously over the capillary with $L_{p}^{A Q P 4}=8.05 \times 10^{-6} \mathrm{~cm} \mathrm{~s}^{-1} \mathrm{mmHg}^{-1}$.

Bar 2. Polarized pattern of AQP4 distribution: $L_{p}^{A Q P 4}=8.05 \times 10^{-6} \mathrm{~cm} \mathrm{~s}^{-1} \mathrm{mmHg}^{-1}$ in the CRA and the COA; $L_{p}^{A P^{4 *}}=6.23 \times 10^{-6} \mathrm{~cm} \mathrm{~s}^{-1} \mathrm{mmHg}^{-1}$ in the CFA. Bar 3. Polarized pattern of AQP4 distribution: $L_{p}^{A Q P 4}=8.05 \times 10^{-6} \mathrm{~cm} \mathrm{~s}^{-1} \mathrm{mmHg}-1$ in the CFA and the COA; $L_{p}^{A Q P 4^{*}}=6.23 \times 10^{-6} \mathrm{~cm} \mathrm{~s}^{-1} \mathrm{mmHg}^{-1}$ in the CRA.

(e) Effect of an increased intracranial pressure on the overall fluid filtration volume. Bar 1

and Bar 2 are obtained with the ICP wave 1 and wave 2 (Figure lb), respectively. (f) The impact of the elevated venous hydrostatic pressure on the overall transcapillary fluid movement. Subscripts 1, 2, 3, 4, 5 stand for the hydrostatic pressure at the venous end of the capillary equal to $15 \mathrm{mmHg}, 15.5 \mathrm{mmHg}$, $16 \mathrm{mmHg}, 17 \mathrm{mmHg}$, and $18 \mathrm{mmHg}$, correspondingly.

capillary length for the human brain is estimated at about $53 \mu \mathrm{m}$ [45]. In the present model, the length of a capillary $L=500 \mu \mathrm{m}$. Taken against the mean capillary dimension, it gives a possibility to estimate transcapillary fluid exchange with the capillaries of length $L^{*}$ with $L>L^{*}$. 
Figure 4c presents water filtration and reabsorption patterns on stepwise reduction of the capillary length starting from either the venular or the arterial end of the capillary. The upper set of the bars demonstrates an increased filtration volumes on the capillary length reduction from the venular end. Reabsorption increases on the capillary length reduction from the arterial end (the set of bars on the negative scale). Variations in the length of a capillary results in establishing different axial hydrostatic pressure profiles. The axial shift of the hydrostatic pressure profiles effects the net driving force, direction, and magnitude of the capillary radial flow rates.

\section{AQP4 polarization in the astrocyte endfeet membrane and the net radial flow rates}

AQP4 presents a rate-limiting step in water movement across the BBB. Lateral distribution of AQP4 in the astrocyte endfeet membrane is region-specific and tends to vary under both normal and pathological conditions $[34,46]$. Polarization of AQP4 in the astrocyte endfeet membrane sheathing a capillary finds its reflection in the region-specific values for the hydraulic conductivity coefficients. This is the way a polarized AQP4 expression may effect fluid movement between blood and the interstitial space.

We modelled the effects of AQP4 polarization by assigning different hydraulic conductivity coefficients $\left(L_{p}^{A Q P 4}=8.05 \times 10^{-6} \mathrm{~cm}\right.$ $\mathrm{mmHg}^{-1} \mathrm{~s}^{-1}$ and $L_{p}^{A Q P 4^{*}}=6.23 \times 10^{-6} \mathrm{~cm} \mathrm{mmHg}^{-1} \mathrm{~s}^{-1}$ ) to the water-exchange areas of the capillary. With AQP4 homogeneously distributed over all areas of the capillary there is established a net positive flux of fluid with the capillary operating in a filtrating mode (Figure $4 \mathrm{~d}$, Bar 1). On assigning $L_{p}^{A Q P^{4^{*}}}$ exclusively to the CFA turns the capillary into a fluid-reabsorbing one (Figure $4 \mathrm{~d}$, Bar 2). With $L_{p}^{A Q P^{*}}$ assigned to the CRA the net water filtration volume is increased by over 2.6 times (Bar 3 ) compared to the homogeneous distribution case (Bar 1).

\section{Elevated intracranial pressure and the net fluid filtration rate}

Animal experiments demonstrate that increasing intracranial pressure slows down CSF production [47]. The simulations carried out with the two pulse waveforms differing by their peak intensities, shown in Figure 1a, demonstrate that elevation of the intracranial pressure by $5 \mathrm{mmHg}$ peak intensity results in the overall fluid filtration decrease by $84 \%$ (Figure $4 \mathrm{e}$ ). This might serve for an explanation for the reduced CSF production.

\section{Elevated venous hydrostatic pressure and the transcapillary fluid exchange}

Elevation of the venous hydrostatic pressure results in an increased filtration (decreased reabsorption) of the water as shown in Figure 4f. It is interesting to observe that elevation of the venous hydrostatic pressure by mere $0.5 \mathrm{mmHg}$ (the first two bars in Figure 4f) brings about a complete reversal of the capillary fluid exchange pattern: the fluid-absorbing mode of the capillary at 15 $\mathrm{mmHg}$ turns into a fluid-filtrating one at $15.5 \mathrm{mmHg}$. The dependence of the transferred fluid volumes on the hydrostatic venous pressure is linear.

\section{Discussion}

The brain fluids present important inner mobile medium closely involved in physiological functions of the brain [14,48]. It is clearly understood at present that the knowledge of fluid/water dynamics in the brain interstitial space together with that of neural circuits is the way to clarify the complex physiology of the brain [1].

The water metabolism disorders manifest themselves in a broad spectrum of cases and present the centre of attention to many research groups focused on finding effective therapeutic means to correct them. The development of predictive mathematical models of brain water metabolism, that would help to explain the fundamental science behind brain physiology and various clinical conditions, becomes very important.

There is a standing controversy at present about the mechanism of brain water metabolism. Emerging new theories challenge the classical theory and put stress on fluid formation and reabsorption at the microvascular level all over brain parenchyma $[8,9,49,50]$. The adherents of the diffusion theory maintain that the laws of diffusion are sufficient to explain the events taking place there [15,16]. Within their concept, the interstitial space is looked upon as "a diffusion barrier" [17]. From the characteristic nanodimentional scale of the interstitial space they have come to a conclusion that the spaces between the cells, within the neuropil, are too narrow to permit any significant bulk flow [51].

The researchers dissatisfied with the diffusion barrier theory suggest fluid convection in the interstitial space $[4,5,7,14,26,50,52]$. However computer simulations, using a conventional no-slip approach, demonstrate that any significant convection there would demand unrealistically high hydrostatic pressure gradients [15]. The situation seems to have reached a stalemate.

An interdisciplinary approach makes it possible to see the issue of fluid movements in the brain interstitial space in a different light. The nanodimentional scale of this space has long been recognized [16,51]. The next logical step would have been to consider fluid movement there as governed by the nanofluidics principles. Indeed, in the nanoconfined spaces the surface hydrodynamic slippage of water brings about an increase of the flow rates that become orders of magnitude higher than those predicted from the no-slip Hagen-Poiseuille's or Darcy's equations [18,21]. The nanofluidic approach has the promise to resolve the current stalemate situation and to open new perspectives in understanding brain physiology and diseases. 
We first introduced a conceptual nanofluidic approach to water movement in the brain interstitial while conducting an MRI clinical research on cerebral water metabolism [53]. In the present model, we view the brain interstitial space as a distinctive nanofluidic domain whose very dimensionality dictates that the slip-flow phenomena should govern fluid dynamics there. This assumption means that water movement in the interstitial space is fast enough to ensure a considerable convectional flow and to shift the overall kinetic control to AQP4.

Experimental data demonstrate that AQP4 is a key molecule controlling water exchange between the blood and the brain parenchyma. The experiments on the AQP4 knockout animals and those with the AQP4 inhibitors strongly support this AQP4 function [33]. In translational medicine, AQP4 is considered a target molecule in the drug therapy of various pathologies, arising from water metabolism disorders, a guide for developing new therapeutic approaches [54-59].

Confronted with the AQP4 water-flow-controlling function, the conventional diffusion-barrier concept becomes a kinetic controversy. The diffusion barrier would be the slowest step in the overall water movement sequence, thus making AQP4 control function redundant. However, this would contradict the vast experimental and clinical data on the AQP4 control function.

The nanofluidic domain concept removes this controversy. It also resolves the problems of the convectional no-slip flow theories that demand high-pressure gradients to ensure convectional water movement through the nanodimentional interstitial space. The nanofluidic approach implies very fast fluid movement in the brain interstitial space, which makes AQP4 the slowest step and puts it in control of the overall fluid flow rate.

The nanofluidic model demonstrates that within brain microvascular network there are found the filtrating and reabsorbing capillaries responsible for water metabolism and whose function depends on the phase of the heart cycle, hydrostatic pressure gradients, capillary length, and AQP4 polarization. These regulatory factors are important for brain fluid homeostasis and physiology.

The simulation results (Figure 4d) demonstrate that altered lateral polarisation of AQP4 in the astrocyte endfeet membrane may have far-reaching consequences for the brain water metabolism. AQP4 content and polarization depend on the brain cortex local physiological activity. Changes in AQP4 subcellular polarization occur in response to various conditions including stroke, Alzheimer's disease, brain tumours and many others pathologies [60,61]. The nanofluidic model describes the ways AQP4 lateral polarization effects brain water metabolism and quantifies its impact. The sensitivity of the brain water metabolism to the changes in the AQP4 lateral polarization underscores the importance of detailed knowledge of AQP4 topography at nano- and microscale in the astrocyte endfeet membrane enveloping a capillary.

The nanofluidic model makes visible the details of the transcapillary water exchange process in relation to the phases of the heart cycle. A distinct oscillatory pattern of fluid movement in the COA deserves a special attention. The oscillatory fluid flux here may serve as a means of communication between the periphery and the central nervous system. The fluid crossing the BBB carries information to the systemic blood about the state of brain metabolism and brings a feedback information from the periphery. The frequency of this exchange is synchronized with the heart cycles.

This feedback exchange within the COA may have important implications for the local neurovascular coupling that requires a close temporal and regional linkage between neural activity and cerebral blood [62]. The shifts in the local functional and metabolic demands of the brain are accompanied by corresponding blood flow responses [45]. Increased capillary flow would necessary result in establishing a new longitudinal pressure profile in a capillary. This may be a way of regulating capillary filtration capacity and switching capillary function from fluid-filtrating to fluid-reabsorbing one. This approach makes it possible to have a glimpse at complex interplay of the capillary fluid exchange function. It might be viewed as a demonstration of how capillarylevel response, in terms of magnitude and direction of radial fluid fluxes, can be attuned to brain homeostatic and physiological demands.

The nanofluidic model may have implications for the translational medicine. The ICP pulsations and intracranial pressure waveforms present much interest in clinical diagnosis of neurological diseases. The model makes it possible to observe the details of the transcapillary fluid exchange in relation the phases of the ICP pulse waveforms generated by the heart activity. It demonstrates the ways the intracranial pulsatility may affect ISF/CSF hydrodynamics and might be useful in solving some experimental and clinical problems in neurology and neurosurgery.

The developed nanofluidic model demonstrates the ways the capillary-level water-exchange response, in terms of the magnitude and direction of radial fluid fluxes, is affected by the AQP4 activity and polarization, the intracranial pressure, the capillary length. It might find its use in elucidation of the pathogenic mechanisms of cerebrovascular disease, brain edema, hydrocephalus, Alzheimer, dementia and others.

The model might be used for development of the AQP4-targeted drug therapy, optimization of the intrathecal drug delivery in the brain tumour therapy, and in the therapy of a broad spectrum of water-metabolism-related disorders. 


\section{Conclusions}

An adopted interdisciplinary approach has made it possible to consider the brain interstitial space as a nanofluidic domain where water movement is governed by the slip-flow principles of nanofluidics. The model allows for a new look at the brain fluid physiology.

In the developed computational model, AQP4 kinetically controls the movement of the interstitial and the cerebrospinal fluid in the brain nanofluidic domain. AQP4 presents a molecular target for a directed action of the drugs that might be used for the therapy of a number of clinical conditions. The model demonstrates good predictability in respect to some clinically relevant cases of water metabolism disorders. It underscores the importance of the cardiocerebral relationship for the brain water metabolism. The model describes a molecular-level approach to a possible control and therapy of the water-metabolism-disorder-related conditions.

\section{Acknowledgements}

The author would like to express his gratitude to the Head of the Biophysics Department of the Belarussian State University Professor S. Cherenkevich, Ph.D. for useful discussions.

The author acknowledges financial support from the National Academy of Sciences of Belarus through grant 3.09-2016-20 of State Research Programme "Convergency-2020".

\section{Conflict of interest declaration}

The author declares no competing financial interests.

\section{Supplementary data}

Supplementary data to this article can be found online at Online Resource.

\section{References}

1. Lei Y, Han H, Yuan F, Javeed A, Zhao Y (2017) The brain interstitial system: Anatomy, modeling, in vivo measurement, and applications. Prog Neurobiol 157: 230-46.

2. Simon MJ, Iliff JJ (2016) Regulation of cerebrospinal fluid (CSF) flow in neurodegenerative, neurovascular and neuroinflammatory disease. Biochim Biophys Acta 1862: 442-51.

3. Orešković D, Klarica M (2014) A new look at cerebrospinal fluid movement. Fluids Barriers CNS 11: 1-16.

4. Titovets E, Nechipurenko N, Griboedova T, Vlasyuk P (2000) Experimental study on brain oxygenation in relation to tissue water redistribution and brain oedema. Acta Neurochir Suppl 76: 279-81.

5. Jessen NA, Munk AS, Lundgaard I, Nedergaard M (2015) The Glymphatic System: A Beginner’s Guide. Neurochem Res 40: 2583-99.

6. Chikly B, and Quaghebeur J (2013) Reassessing cerebrospinal fluid (CSF) hydrodynamics: a literature review presenting a novel hypothesis for CSF physiology. J Bodyw Mov Ther 17: 344-54.

7. Titovets E (2007) Aquaporins of Man and Animals. Basic and Clinical Aspects. Izdatelski Dom "Belaruskaya Nauka”.

8. Buishas J, Gould IG, Linninger AA (2014) A computational model of cerebrospinal fluid production and reabsorption driven by Starling forces. Croat Med J 55: 481-97.

9. Miyajima M, Arai H (2015) Evaluation of the Production and Absorption of Cerebrospinal Fluid. Neurol Med Chir (Tokyo) 55: 647-56.

10. Silva AC, Zhang W, Williams DS, Koretsky AP (1997) Estimation of Water Extraction Fractions in Rat Brain Using Magnetic Resonance Measurement of Perfusion with Arterial Spin Labeling. Magn Reson Med 35: 58-68.

11. Cutler R, Page L, Galicich J, Watters GV (1968) Formation and absorption of cerebrospinal fluid in man. Brain 91: 707-20.

12. Nicholson C (2007) Modeling Brain Extracellular Space from Diffusion Data. Diffu Fundament 6: 75.1-75.15.

13. Kamali-Zare P, Nicholson C (2013) Brain Extracellular Space: Geometry, Matrix and Physiological Importance. Basic Clin Neurosci. 4: 282-86.

14. Abbott NJ (2004) Evidence for bulk flow of brain interstitial fluid: significance for physiology and pathology. Neurochem Int 45: 545-52.

15. Jin BJ, Smith AJ, Verkman AS (2016) Spatial model of convective solute transport in brain extracellular space does not support a "glymphatic" mechanism. J Gen Physiol 148: 489-501.

16. Sykova E, Nicholson C (2008) Diffusion in brain extracellular space. Physiol Rev 88: 1277-340.

17. Nicholson C, Kamali-Zare P, Tao L (2011) Brain Extracellular Space as a Diffusion Barrier. Comput Vis Sci 14: 309-25.

18. Abgrall P, Nguyen NT (2009) Nanofluidics, Artech House, Boston, USA.

19. Mitra S, Chakraborty S (2011) Microfluidics and Nanofluidics Handbook. Chemistry, Physics, and Life Science Principles. CRC Press, Taylor \& Francis Group, UK.

20. Eijkel J, van den Berg A (2005) Nanofluidics: what is it and what can we expect from it? Microfluid Nanofluidics 1: 249-67.

21. Sparreboom W, Van den Berg A, Eijkel JCT (2010) Transport in nanofluidic systems: a review of theory and applications. New J Phys 12: 1-23.

22. Bocquet L, Charlaix E (2010) Nanofluidics, from bulk to interfaces. Chem Soc Rev 39: 1073-95.

23. Soltani M, Chen P (2013) Numerical Modeling of Interstitial Fluid Flow Coupled with Blood Flow through a Remodeled Solid Tumor Microvascular Network. PLoS One 8: 1-18. 
24. Smith AJ, Yao X, Dix JA, Jin BJ, Verkman AS (2017) Test of the 'glymphatic' hypothesis demonstrates diffusive and aquaporin-4-independent solute transport in rodent brain parenchyma. Elife 6: e27679.

25. Godin AG1, Varela JA, Gao Z, Danné N, Dupuis JP, et al. (2017) Single-nanotube tracking reveals the nanoscale organization of the extracellular space in the live brain. Nat Nanotechnol 12: 238-43.

26. Igarashi H, Tsujita M, Kwee IL, Nakada T (2014) Water influx into cerebrospinal fluid is primarily controlled by aquaporin-4, not by aquaporin-1: 17O JJVCPE MRI study in knockout mice. Neuroreport 25: 39-43.

27. Xu M, Xiao M, Li S, Yang B (2017) Aquaporins in Nervous System. Adv Exp Med Biol 969: 81-103.

28. Filippidis AS, Carozza RB, Rekate HL (2016) Aquaporins in Brain Edema and Neuropathological Conditions. Int J Mol Sci 18 : E55.

29. Desai B, Hsu Y, Schneller B, Hobbs JG, Mehta AI, et al. (2016) Hydrocephalus: the role of cerebral aquaporin-4 channels and computational modeling considerations of cerebrospinal fluid. Neurosurg Focus 41: E8.

30. Fallier-Becker P, Sperveslage J, Wolburg H, Noell S (2011) The impact of agrin on the formation of orthogonal arrays of particles in cultured astrocytes from wild-type and agrin-null mice. Brain Res 1367: 2-12.

31. Assentoft M, Larsen BR, MacAulay N (2015) Regulation and Function of AQP4 in the Central Nervous System. Neurochem Res 40: 2615-27.

32. Furman CS, Gorelick-Feldman DA, Davidson KG, Yasumura T, Neely JD, et al. (2003) Aquaporin-4 square array assembly: Opposing actions of M1 and M23 isoforms. Proc Natl Acad Sci U S A 100: 13609 -13614.

33. Haj-Yasein NN, Vindedal GF, Eilert-Olsen M, Gundersen GA, Skare O et al. (2011) Glial-conditional deletion of aquaporin-4 (Aqp4) reduces blood-brain water uptake and confers barrier function on perivascular astrocyte endfeet. Proc Natl Acad Sci U S A 108: 17815-20.

34. Nagelhus EA, Ottersen OP (2013) Physiological roles of aquaporin-4 in brain. Physiol Rev 93: 1543-62.

35. Wagshul ME, Chen JJ, Egnor MR, McCormack EJ, Roche PE (2006 ) Amplitude and phase of cerebrospinal fluid pulsations: experimental studies and review of the literature. J Neurosurg 104: 810-9.

36. Wagshul ME, Eide PK, Madsen JR (2011) The pulsating brain: A review of experimental and clinical studies of intracranial pulsatility. Fluids Barriers CNS 8: $1-23$.

37. Bhadelia RA, Bogdan AR, Kaplan RF, Wolpert SM (1997) Cerebrospinal fluid pulsation amplitude and its quantitative relationship to cerebral blood flow pulsations: a phase-contrast MR flow imaging study. Neuroradiology 39: 258-64.

38. Oldendorf WH (1970) Measurement of brain uptake of radiolabeled substances using a tritiated water internal standard. Brain Research 24: $372-6$.

39. Brinker T, Stopa E, Morrison J, Klinge P (2014) A new look at cerebrospinal fluid circulation. Fluids Barriers CNS 11: 10.

40. Kao YH, Guo WY, Liou AJ, Chen TY, Huang CC, et al. (2013) Transfer function analysis of respiratory and cardiac pulsations in human brain observed on dynamic magnetic resonance images. Comput Math Methods Med 2013: 157040.

41. Friedman MH (1986) Principles and Models of Biological Transport, Springer-Verlag Berlin Heiderberg GmbH, Germany.

42. Greitz D, Franck A, Nordell B (1993) On the pulsatile nature of intracranial and spinal CSF-circulation demonstrated by MR imaging. Acta Radiol 34: 321-8.

43. Hudetz AG, Greene AS, Fehér G, Knuese DE, Cowley AW Jr (1993) Imaging System for Three-Dimensional Mapping of Cerebrocortical Capillary Networks in Vivo. Microvasc Res 46: 293-309.

44. Ivanov KP, Kalinina MK, Levkovich YI (1981) Blood flow velocity in scapillaries of brain and muscles and its physiological significance. Microvasc Res 22: 143-55.

45. Pawlik G, Rackl A, Bing RJ (1981) Quantitative capillary topography and blood flow in the cerebral cortex of cats: an in vivo microscopic study. Brain Res 208: 35-58.

46. Aoyama M, Kakita H, Kato S, Tomita M, Asai K (2012) Region-specific expression of a water channel protein, aquaporin 4, on brain astrocytes. J Neurosci Res 90: $2272-80$.

47. Maraković J, Oresković D, Rados M, Vukić M, Jurjević I (2010) Effect of osmolarity on CSF volume during ventriculo-aqueductal and ventriculo-cisternal perfusions in cats. Neurosci Lett 484: 93-7.

48. Agnati LF, Guidolin D, Guescini M, Genedani S, Fuxe K (2010) Understanding wiring and volume transmission. Brain Res Rev 64: 137-59.

49. Orešković D, Radoš M, Klarica M (2017) New Concepts of Cerebrospinal Fluid Physiology and Development of Hydrocephalus. Pediatr Neurosurg 52: 417-25.

50. Titovets EP, Stepanova TS (2004) Conceptual mathematical model for convective mechanism of brain cortex oxygenation. News Biomed Sci 2: 127-34.

51. Thorne RG, Nicholson C (2006) In vivo diffusion analysis with quantum dots and dextrans predicts the width of brain extracellular space. Proc Natl Acad Sci U S A 103: 5567-72.

52. Bobo RH, Laske DW, Akbasak A, Morrison PF, Dedrick RL, et al. (1994) Convection-enhanced delivery of macromolecules in the brain. Proc Natl Acad Sci U S A 91: 2076-80.

53. Titovets E (2015) Research on the cerebral water metabolism disorders using functional MRI visualization [in Russian]. Proceedings of the National Academy of Sciences of Belarus Medical Series 1: 65-72.

54. Papadopoulos MC, Verkman AS (2008) Potential utility of aquaporin modulators for therapy of brain disorders. Prog Brain Res 170: 589-601.

55. Verkman AS, Smith AJ, Phuan PW, Tradtrantip L, Anderson MO (2017) The aquaporin-4 water channel as a potential drug target in neurological disorders. Expert Opin Ther Targets 21: 1161-70.

56. Badaut J, Fukuda AM, Jullienne A, Petry KG (2014) Aquaporin and brain diseases. Biochim Biophys Acta 1840: $1554-65$.

57. Siegel, G.J. (2012) Therapy of Brain Edema Potential Pharmacologic Regulation of Aquaporin 4, American Society for Neurochemistry. Elsevier, USA.

58. Previch LE, Ma L, Wright JC, Singh S, Geng X, et al. (2016) Progress in AQP Research and New Developments in Therapeutic Approaches to Ischemic and Hemorrhagic Stroke. Int J Mol Sci 17: 1-16.

59. Lan YL, Chen JJ, Hu G, Xu J, Xiao M, et al. (2017) Aquaporin 4 in astrocytes is a target for therapy in Alzheimer's disease. Curr Pharm Des 23: $4948-957$.

60. Crane JM, Van Hoek AN, Skach WR, Verkman AS (2008) Aquaporin-4 dynamics in orthogonal arrays in live cells visualized by quantum dot single particle tracking. Mol Biol Cell 19: 3369-78.

61. Wolburg H, Wolburg-Buchholz K, Fallier-Becker P, Noell S, Mack AF (2011) Structure and Functions of Aquaporin-4-Based Orthogonal Arrays of Particles. Int Rev Cell Mol Biol 287: 1-41. 
62. O’Herron P, Chhatbar PY, Levy M, Shen Z, Schramm AE, et al. (2016) Neural correlates of single-vessel haemodynamic responses in vivo. Nature 534: 378-82.

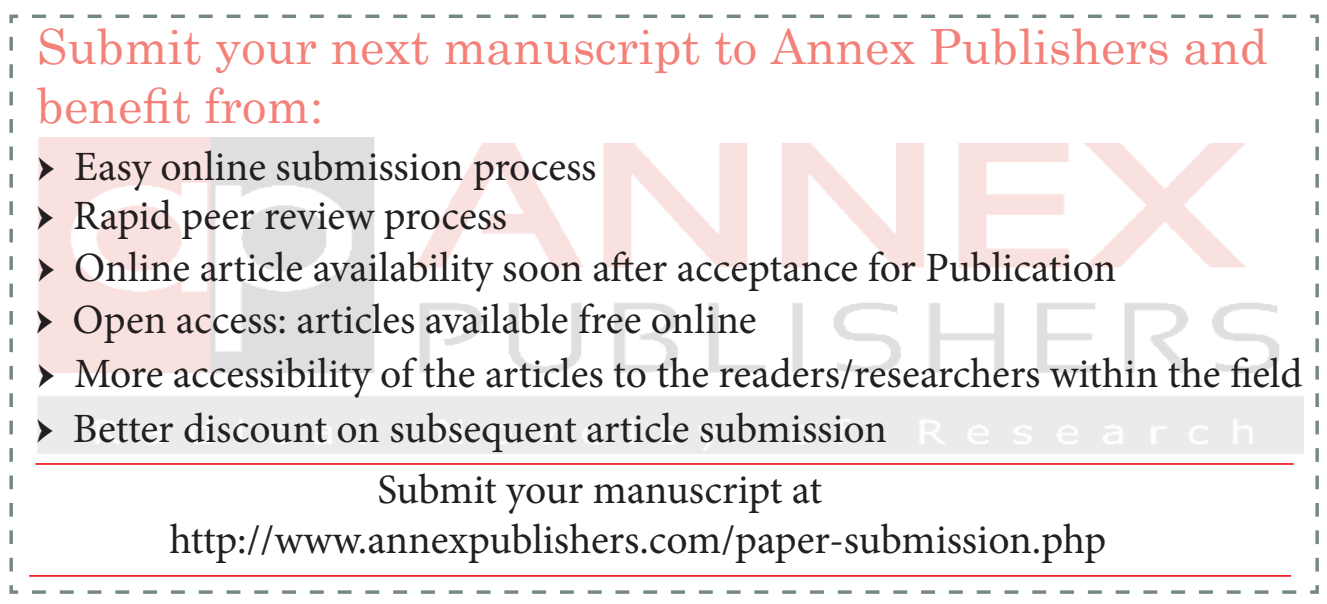

